\title{
CCNE1 copy-number gain and overexpression identify ovarian clear cell carcinoma with a poor prognosis
}

Ayse Ayhan ${ }^{1,2,3,4}$, Elisabetta Kuhn ${ }^{5}$, Ren-Chin $\mathrm{Wu}^{6}$, Hiroshi Ogawa ${ }^{2}$, Asli Bahadirli-Talbott ${ }^{1}$, Tsui-Lien $\mathrm{Mao}^{7}$, Haruhiko Sugimura ${ }^{3}$, Ie-Ming Shih ${ }^{1,8}$ and Tian-Li Wang ${ }^{1,8}$

${ }^{1}$ Department of Pathology, Johns Hopkins Medical Institutions, Baltimore, MD, USA; ${ }^{2}$ Department of Pathology, Seirei Mikatahara Hospital, Hamamatsu, Japan; ${ }^{3}$ Department of Tumor Pathology, Hamamatsu University School of Medicine, Hamamatsu, Japan; ${ }^{4}$ Department of Molecular Pathology, Hiroshima University School of Medicine, Hiroshima, Japan; ${ }^{5}$ Pathology Unit, Arcispedale S. Maria Nuova-IRCCS, Reggio Emilia, Italy; ${ }^{6}$ Department of Pathology, Chang Gung Memorial Hospital and Chang Gung University College of Medicine, Taoyuan, Taiwan; ${ }^{7}$ Department of Pathology, National Taiwan University College of Medicine, Taipei City, Taiwan and ${ }^{8}$ Department of Gynecology and Obstetrics, Johns Hopkins Medical Institutions, Baltimore, MD, USA

Ovarian clear cell carcinoma is a unique type of ovarian cancer, often derived from endometriosis, and advancedstage disease has a dismal prognosis primarily due to the resistance to conventional chemotherapy. Previous studies have shown frequent somatic mutations in ARID1A, PIK3CA, hTERT promoter, and amplification of ZNF217; however, the molecular alterations that are associated with its aggressiveness remain largely unknown. This study examined and compared cyclin E1 expression in endometriosis-related ovarian tumors, with the aim of determining the relationship between $h T E R T$ mutations and ARID1A expression and evaluating the effects of these molecular alterations on patient survival. We performed immunohistochemistry on 207 tumors [clear cell carcinoma $(n=120)$, endometrioid carcinoma $(n=49)$, and seromucinous tumors $(n=38)$ ], followed by two-color fluorescence in situ hybridization $(n=88)$ and compared with ARID1A expression and $h T E R T$ promoter mutations in the same samples. Cyclin E1 overexpression and CCNE1 copy-number gain occurred in $23.3 \%$ and $14.8 \%$ of ovarian clear cell carcinomas, respectively, but they were not detected in any of the other endometriosis-related tumors. All cases with CCNE1 copy-number gain demonstrated an intense cyclin E1 immunoreactivity $(P<0.001)$. Cyclin E1 overexpression was positively correlated with $h T E R T$ promoter mutations $(P=0.01)$, but not with the loss of ARID1A expression. A multivariate analysis revealed that CCNE1 overexpression predicts poor overall survival, even after adjusting for stage and age. Specifically, CCNE1 overexpression and copy-number gain were both correlated with a poor outcome in patients with stage I disease. Moreover, the subset with CCNE1 overexpression and ARID1A retention demonstrated the worst outcome. Our findings suggest that gene copy-number gain and upregulation of CCNE1 occur in ovarian clear cell carcinoma and are associated with a worse clinical outcome, dictating the survival of early-stage patients, and that these molecular alterations are unique to clear cell carcinoma among different types of endometriosis-related ovarian neoplasms.

Modern Pathology (2017) 30, 297-303; doi:10.1038/modpathol.2016.160; published online 21 October 2016

Ovarian cancer is a heterogeneous disease that comprises at least five histological subtypes, which are characterized by distinct clinicopathological and

Correspondence: Adjunct Professor $\mathrm{Dr}$ A Ayhan, $\mathrm{MD}, \mathrm{PhD}$ or Professor T-L Wang, PhD, Department of Pathology, Johns Hopkins Medical Institutions, 1550 Orleans Street, Baltimore, MD 21231, USA. E-mail: ayseayhanjp@gmail.com or tlw@jhmi.edu

Received 11 May 2016; revised 29 July 2016; accepted 30 July 2016; published online 21 October 2016 molecular features as well as by their cellular origins. ${ }^{1}$ Among them, ovarian clear cell carcinoma, endometrioid carcinoma, and seromucinous neoplasms represent a unique group of diseases because they are often associated with endometriosis. Because most of these cancers arise from preexisting ovarian endometriotic cysts, it has been suggested that the inflammatory and oxidative stress associated with the endometriosis microenvironment promote mutagenesis and facilitate tumor 
development. ${ }^{2}$ Compared with other types of ovarian carcinoma, endometriosis-related ovarian cancers are characterized molecularly by aberrations in the PI3K-AKT pathway and frequent somatic mutations involving $A R I D 1 A$, a tumor suppressor gene that participates in the SWI/SNF chromatin remodeling complex. ${ }^{3}$ Although endometriosis-related neoplasms share several common features, they also differ in many respects. For example, compared with endometrioid carcinoma, clear cell carcinoma is characterized by unique histopathological characteristics, resistance to platinum-based chemotherapy, presence of activating mutations in the hTERT promoter, and expression of specific biomarkers including $\mathrm{HNF}-1 \beta$, Napsin A, and $\alpha$-methylacyl-coenzyme A racemase (AMACR, P504S). ${ }^{4-7}$ Genetically engineered mouse models have demonstrated that the co-deletion of the ARID1A and $P T E N$ genes induces ovarian endometrioid carcinoma in the murine ovary, whereas the deletion of ARID1A and the expression of a PIK3CA activating mutant generate ovarian clear cell-like tumors. ${ }^{8,9}$

In a previous study, we identified DNA copynumber gain at the CCNE1 locus in a small set of ovarian clear cell carcinomas. ${ }^{10}$ CCNE1 encodes the cyclin E1 protein of the highly conserved 'cyclin' family of proteins, which are involved in cell cycle regulation. Cyclin E1 forms a complex with the regulatory subunit, cyclin-dependent kinase-2 (Cdk2), and promotes transition of the G1 to $\mathrm{S}$ phase of the cell cycle. Although normal cells maintain strict control of cyclin E1 activity, cancer cells can exploit the upregulation of this protein as a way to promote tumor cell replication. Several studies have reported that CCNE1 gene amplification or protein upregulation is associated with higher tumor grades and with a worse clinical outcome in a variety of cancers. ${ }^{11-13}$ For example, increased DNA copy number and overexpression of CCNE1 are observed in uterine serous carcinoma, a high-grade uterine cancer, but not in the more indolent uterine endometrioid carcinoma. ${ }^{14-16}$ Similarly, CCNE1 amplification characterizes ovarian high-grade, but not low-grade, serous carcinomas. ${ }^{17-19}$ To further explore the significance of CCNE1 in endometriosisrelated ovarian cancers, we performed immunohistochemistry and fluorescence in situ hybridization (FISH) analyses of CCNE1.

\section{Materials and methods}

\section{Tissue Samples}

The paraffin-embedded tumor tissues were obtained from the Seirei Mikatahara Hospital and from the file of The Johns Hopkins Hospital. In all, after excluding those with neoadjuvant chemotherapy, 120 anonymous cases of ovarian clear cell carcinoma (96 cases from tissue microarrays and 24 from whole sections), 49 cases of ovarian endometrioid carcinoma (31 from tissue microarrays and 18 from whole sections), and 38 cases of seromucinous neoplasm (12 malignant, the remaining were borderline) were collected between 1995 and 2013. Ninetyfour patients with ovarian clear cell carcinoma underwent clinical follow-up for up to 175 months, and all of the patients who were followed-up were from East Asian populations. All samples were anonymized, and the study was approved by the institutional review boards of each participating hospital. The selection criteria were based on the availability of archived tissue, tumors from patients receiving similar treatments, and pre-existing followup data rather than on other clinical or demographic characteristics.

\section{Immunohistochemistry}

Immunohistochemistry was performed to assess the expression levels of cyclin E1 in paraffin-embedded tissue sections that were obtained from 120 ovarian clear cell carcinomas, 49 endometrioid carcinomas, and 38 seromucinous neoplasms. In brief, after deparaffinization and rehydration, the slides were placed in citrate buffer (Vector Laboratories, Burlingame, CA, USA) for antigen retrieval. An anti-cyclin E1 antibody (Sigma-Prestige, St Louis, MO, USA) was then applied at a dilution of 1:300. After incubation with the primary antibody at room temperature for $2 \mathrm{~h}, 3,3^{\prime}$-diaminobenzidine was used to develop immunoreactivity, which was detected by the EnVision+System (Dako, Carpinteria, CA, USA). The following simple dichotomous system was used for the evaluation of CCNE1 expression: diffuse and intense immunoreactivity in $>80 \%$ of tumor cells were considered positive (abnormal or overexpressed), which was based on the agreement of three pathologists (AA, EK, and IMS). Observers were blind to survival or FISH data.

\section{Fluorescence In Situ Hybridization}

DNA copy number at the CCNE1 locus was determined by FISH in 88 cases of ovarian clear cell carcinoma (74 on tissue microarray and 14 on whole tissue sections). In brief, $4-\mu \mathrm{m}$ thick sections were deparaffinized in xylene, hydrated through graded ethanol, and incubated with proteinase $\mathrm{K}$ at $37^{\circ} \mathrm{C}$ for $30 \mathrm{~min}$. After washing in $2 \times$ Aniara saline-sodium citrate, the slides were placed in a denaturation solution $(70 \%$ formamide $/ 2 \times$ saline-sodium citrate at $75^{\circ} \mathrm{C}$ for $5 \mathrm{~min}$ ) and rinsed in $2 \times$ saline-sodium citrate. CCNE1/CEN19p FISH probe (cat\# FG0013, Abnova, Taipei, Taiwan) was applied to the slides and cover slipped. DNA was denatured thorough incubation for $15 \mathrm{~min}$ at $80^{\circ} \mathrm{C}$ and hybridization was performed at $37^{\circ} \mathrm{C}$ for $20-24 \mathrm{~h}$. After washing for $20 \mathrm{~min}$ in $1.5 \mathrm{~mol} / \mathrm{l}$ urea in $0.2 \times$ saline-sodium citrate, slides were drained, dehydrated through graded ethanol, air-dried, counterstained with 4'-6-diamidino-2-phenylindole (cat\# P-36931, 
Invitrogen, Eugene, Oregon, USA) at a concentration of $500 \mathrm{ng} / \mathrm{ml}$ (Sigma) for $3 \mathrm{~min}$ at room temperature, washed in water, mounted with ProLong Gold Antifade mounting medium (Molecular Probes, Eugene, OR, USA), and imaged. ${ }^{15,19}$

\section{Image Analysis}

Photomicrographs from individual cases were captured using a Nikon 50i epifluorescence microscope equipped with fluorescence excitation/emission filters for different fluorophores (Omega Optical) used for CCNE1 FISH. Grayscale images were merged using Nikon NIS-Elements software with an attached Photometrics Cool snap EZ digital camera. The CCNE1 copy number per cell was analyzed in at least 50 nonoverlapping nuclei. Tumor cell nuclei were considered to have copy-number gain if the ratio of CCNE1 to centromeric probe (CEP19) was $\geq 2$ in $>20 \%$ of the analyzed tumor cells (amplification) or if $\geq 4$ CCNE1 copies were observed in $\geq 40 \%$ of tumor cells (high polysomy). ${ }^{15,19}$

\section{Statistical Analysis}

Disease-specific overall survival of patients with ovarian clear cell carcinoma with and without cyclin E1 expression and CCNE1 copy-number gain were compared using the Kaplan-Meier method and the Cox proportional hazards model. This was followed by univariate (log-rank rest) and multivariate (Wald test) analyses to determine the significance. The variables examined were stage of the disease, age of the patient, CCNE1 copy-number gain, cyclin E1 overexpression, ARID1A loss, and the presence of somatic mutations in the hTERT promoter. Comparisons of CCNE1 overexpression/copy-number gain and hTERT mutation or ARID1A status were performed using the two-tailed Fisher's exact test. A P-value of 0.05 or less was considered statistically significant.

\section{Results}

The purposes of our study were to evaluate cyclin E expression in endometriosis-related ovarian neoplasms, to assess the relationship of CCNE1 status with other molecular characteristics, and to determine the clinical significance of these molecular alterations. Among 120 ovarian clear cell carcinomas, 28 $(23.3 \%)$ showed cyclin E1 protein overexpression. Fourteen clear cell carcinomas were found to have adjacent normal-appearing endometriotic cysts that demonstrated histologic continuity, but none of which overexpressed CCNE1 (Data not shown). Among 49 ovarian endometrioid carcinomas and 38 seromucinous tumors, none displayed cyclin E1 protein overexpression. To investigate whether cyclin E1 overexpression was caused by gene copy-number gain, we performed a CCNE1 dual-color FISH assay. Indeed,
$13(14.8 \%)$ of 88 clear cell carcinomas available for FISH analysis displayed gene copy-number gain (Figure 1a). Most clear cell carcinomas (7/13) with CCNE1 copy-number gain revealed a CCNE1/CEP19 ratio of 2.0-2.9 (low level gain), whereas 3 carcinomas displayed a $C C N E 1 / C E P 19$ ratio $\geq 3$ (high level gain). Three cases showed high polysomy, that is, parallel increase of CCNE1 copy number and CEP19 probe. All 13 clear cell carcinomas with CCNE1 copy-number gain showed cyclin E1 protein overexpression; therefore, CCNE1 copy-number gain and cyclin E1 overexpression were significantly correlated $(P<0.001$, two-tailed Fisher's exact test). Among 20 cases with positive cyclin E1 staining, 7 did not qualify as CCNE1 copy-number gain by FISH using above criteria. CCNE1 FISH results and cyclin E1 expression status are summarized in Table 1. Based on re-evaluation by hematoxylin and eosin staining, clear cell carcinomas with CCNE1 gain or overexpression did not show any unique morphological characteristics.

Survival analysis in 94 cases with complete followup demonstrated that cyclin E1 overexpression was significantly associated with poor overall survival, even after adjustment for clinical stage and patient age (age $<60$ vs $\geq 60$ years) in the multivariate analysis. Univariate and multivariate overall survival analysis by the Cox proportional hazards model is presented in Table 2 and is illustrated in Figure 1b. Specifically, shorter not only was cyclin E1 overexpression correlated with shorter overall survival when all stages were considered but both copy-number gain and/or overexpression were significantly associated with poor survival of patients with stage I disease (Table 2; Figure 1c). As both gain and overexpression were associated with poor prognosis, we further determined whether immunohistochemistry or FISH or both would be better to identify clinically relevant subsets. There was no statistical difference in terms of survival between those cases 'with overexpression and copy-number gain' and 'with overexpression without copy-number gain' for both all stages $(P=0.1)$ and stage $1 \quad(P=0.82$; Figures $1 \mathrm{~b}$ and $\mathrm{c})$ although the case numbers were low.

Because the loss of ARID1A expression due to inactivating mutations is the most common molecular alteration known to date in ovarian clear cell carcinoma ${ }^{20}$ and because hTERT mutations are specific to this type of ovarian cancer, we determined whether an association was present between these two molecular alterations and CCNE1 upregulation. ARID1A expression and hTERT promoter mutation status in these cases were previously reported by us and were available for evaluation along with CCNE1 status in 65 and 64 overlapping cases, respectively. ${ }^{21,22}$ The results demonstrated that cases with CCNE1 upregulation typically did not harbor mutations in the hTERT promoter $(P=0.01$ and $P=0.04$; Table 3$)$. On the contrary, no significant association was observed between CCNE1 upregulation and loss of ARID1A expression. Interestingly, patients with CCNE1 upregulation and 


\section{a}

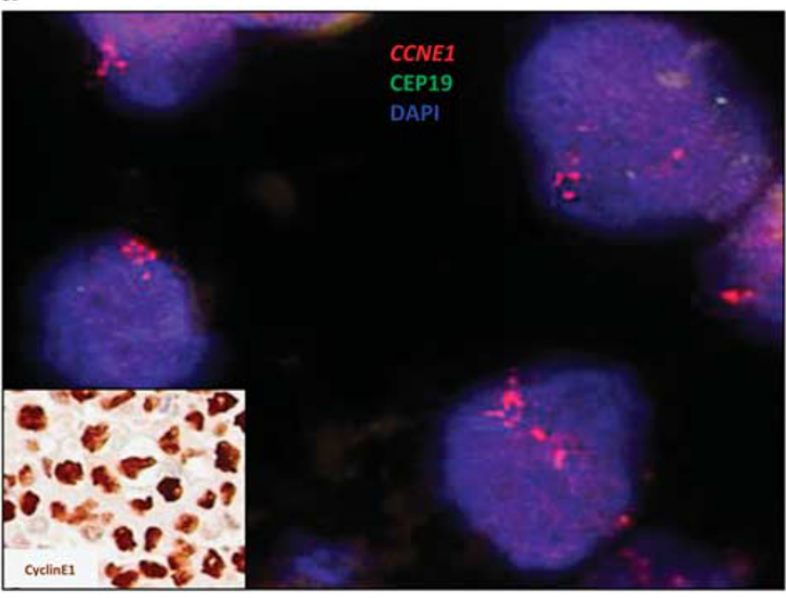

b Overall Survival in Ovarian Clear Cell Carcinoma

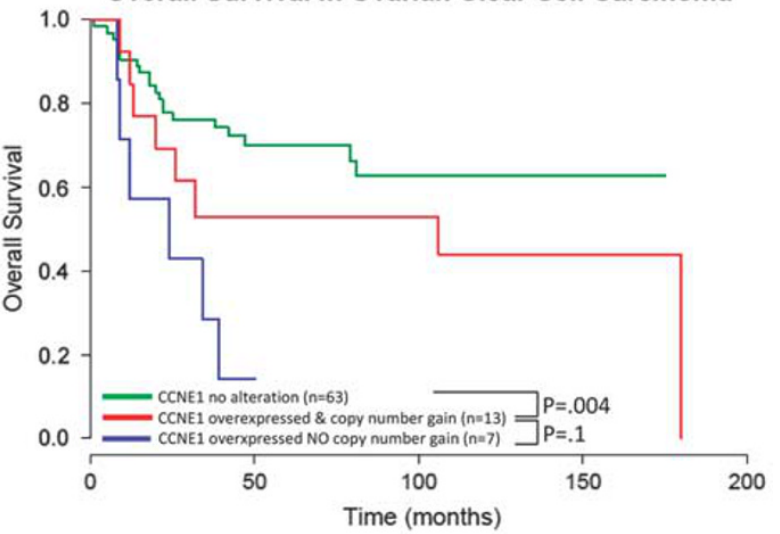

C Overall Survival in Stage I Ovarian Clear Cell Carcinoma

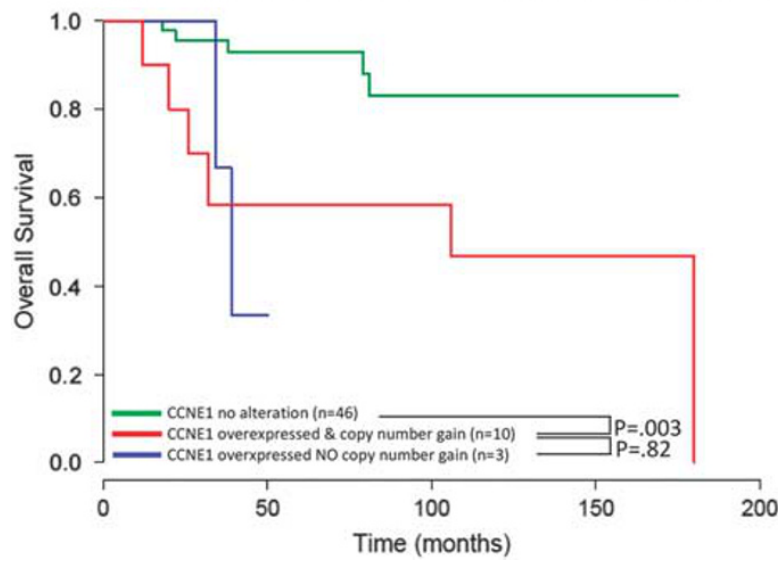

Figure 1 All clear cell carcinomas with CCNE1 copy-number gain have cyclin E1 overexpression. A strong correlation was observed between CCNE1 copy-number gain and cyclin E1 overexpression, $P<0.001$. Increased DNA copy number at the CCNE1 locus by FISH; diffuse and strong CCNE1 expression by immunohistochemistry (inset) (a). CCNE1 overexpression and/or copy-number gain was correlated with overall survival. $P=0.004$ (b). Cyclin E1 overexpression and/or CCNE1 copy-number gain predicted poor survival of patients with stage I clear cell carcinoma $(P=0.003)(\mathbf{c})$. Both overexpression and copy-number gain related to poor prognosis and there was no statistical difference in terms of their contribution to survival in both overall and stage 1 cases (b and $\mathbf{c}$, $P=0.1$ and $P=0.82$ ).
Table 1 Correlation between CCNE1 copy number by fluorescence in situ hybridization and Cyclin E1 immunohistochemistry in 88 ovarian clear cell carcinomas

\begin{tabular}{lccc}
\hline & $\begin{array}{c}\text { CCNE1 no } \\
\text { copy-number } \\
\text { gain }\end{array}$ & $\begin{array}{c}\text { CCNE1 } \\
\text { copy-number } \\
\text { gain }\end{array}$ & Total \\
\hline $\begin{array}{l}\text { Negative cyclin E1 IHC } \\
\text { Positive cyclin E1 IHC }\end{array}$ & $\begin{array}{c}68(77 \%) \\
(8 \%)\end{array}$ & 0 & $68(77 \%)$ \\
\hline
\end{tabular}

$P<0.001$, two-tailed Fisher's exact test.

ARID1A retention had the worst overall survival compared with patients in the other groups (Figure 2). We did not observe any relationship between both ARID1A expression and hTERT promoter mutations with overall survival (data not shown).

\section{Discussion}

Clear cell carcinomas belong to a specific group of ovarian tumors that are frequently associated with endometriosis, especially the lesions that present as ovarian endometriotic cysts (also known as endometriomas). Endometriosis-related ovarian neoplasms include clear cell carcinoma, endometrioid carcinoma, and, less frequently, seromucinous tumors. In this study, we demonstrated that CCNE1 gene copynumber gain and cyclin E1 overexpression are specific to ovarian clear cell carcinoma compared with other types of endometriosis-related neoplasms. In addition, women whose clear cell carcinomas display cyclin E1 overexpression have a shorter overall survival. Remarkably, when patients with stage 1 clear cell carcinoma were analyzed, CCNE1 upregulation indicated a poor prognosis. This is significant because clear cell carcinomas can follow an aggressive clinical course even for those who initially present with early-stage disease. Furthermore, patients with clear cell carcinomas with cyclin E1 overexpression and no concomitant loss of ARID1A expression exhibit the worst clinical outcome. The above results, if they can be confirmed in larger cohorts, may have an impact on the management of patients with clear cell carcinoma.

Ovarian clear cell carcinoma has been traditionally classified as a type I ovarian neoplasm because most patients with this subtype present at early stages and also because these tumors arise from endometriotic cysts or adenofibromas in a stepwise fashion. In addition, these tumors harbor molecular genetic changes, including somatic mutations in ARID1A and PIK3CA, which are commonly shared by other type I ovarian cancers; however, these tumors do not frequently have TP53 mutations. ${ }^{1}$ The finding that CCNE1 copy-number gain in clear cell carcinoma, but not in other type I neoplasms, 
Table 2 Univariate and multivariate overall survival analysis for patients of all stages and for those with stage 1 ovarian clear cell carcinoma by the Cox proportional hazards model

\begin{tabular}{|c|c|c|c|c|c|c|c|c|c|}
\hline \multirow{3}{*}{ Cancer stage } & \multirow{3}{*}{ Variables } & \multicolumn{4}{|c|}{ Univariate analysis } & \multicolumn{4}{|c|}{ Multivariate analysis } \\
\hline & & \multirow{2}{*}{$H R$} & \multicolumn{2}{|c|}{$95 \% C I$} & \multirow{2}{*}{ P-value } & \multirow{2}{*}{$H R$} & \multicolumn{2}{|c|}{$95 \% C I$} & \multirow{2}{*}{ P-value } \\
\hline & & & $L$ & $H$ & & & $L$ & $H$ & \\
\hline \multirow[t]{6}{*}{ All stages } & CCNE1 copy-number gain & 1.42 & 0.61 & 3.29 & 0.41 & - & - & - & - \\
\hline & Cyclin E1 overexpression & 2.16 & 1.11 & 4.18 & 0.02 & 4 & 1.42 & 11.3 & 0.009 \\
\hline & Stage & 3.29 & 2.39 & 4.52 & $<0.001$ & 4.12 & 2.45 & 6.92 & $<0.001$ \\
\hline & Age $>60$ years & 3.65 & 1.7 & 7.83 & $<0.001$ & 6.62 & 1.82 & 24.2 & 0.004 \\
\hline & ARID1A loss & 0.72 & 0.31 & 1.67 & 0.44 & 0.68 & 0.25 & 1.85 & 0.45 \\
\hline & hTERT mutation & 1.15 & 0.42 & 3.13 & 0.78 & 0.55 & 0.14 & 2.15 & 0.39 \\
\hline \multirow[t]{5}{*}{ Stage I } & CCNE1 copy-number gain & 3.77 & 1.18 & 12.1 & 0.02 & 5.38 & 1.24 & 23.4 & 0.02 \\
\hline & Cyclin E1 overexpression & 5.27 & 1.67 & 16.7 & 0.002 & - & - & - & - \\
\hline & Age $>60$ years & 2.67 & 0.33 & 21.3 & 0.34 & 6.6 & 0.61 & 71.3 & 0.12 \\
\hline & ARID1A loss & 0.61 & 0.18 & 2.1 & 0.44 & 0.43 & 0.09 & 1.89 & 0.26 \\
\hline & hTERT mutation & 1.75 & 0.45 & 6.82 & 0.41 & 0.57 & 0.1 & 3.08 & 0.51 \\
\hline
\end{tabular}

Statistically significant $P$-values are depicted in bold.

Table 3 Correlation between hTERT mutations and CCNE1 status in ovarian clear cell carcinomas

\begin{tabular}{lccc}
\hline & Total & hTERT wild type & hTERT mutation \\
\hline Negative cyclin E1 IHC & $45(69 \%)$ & $38(58 \%)$ & $7(11 \%)$ \\
Positive cyclin E1 IHC & $20(31 \%)$ & $12(19 \%)$ & $8(12 \%)$ \\
CCNE1 no copy-number gain & $51(78 \%)$ & $43(66 \%)$ & $8(12 \%)$ \\
CCNE1 copy-number gain & $14(22 \%)$ & $7(11 \%)$ & $7(11 \%)$ \\
\hline
\end{tabular}

Statistically significant $P$-values are depicted in bold.
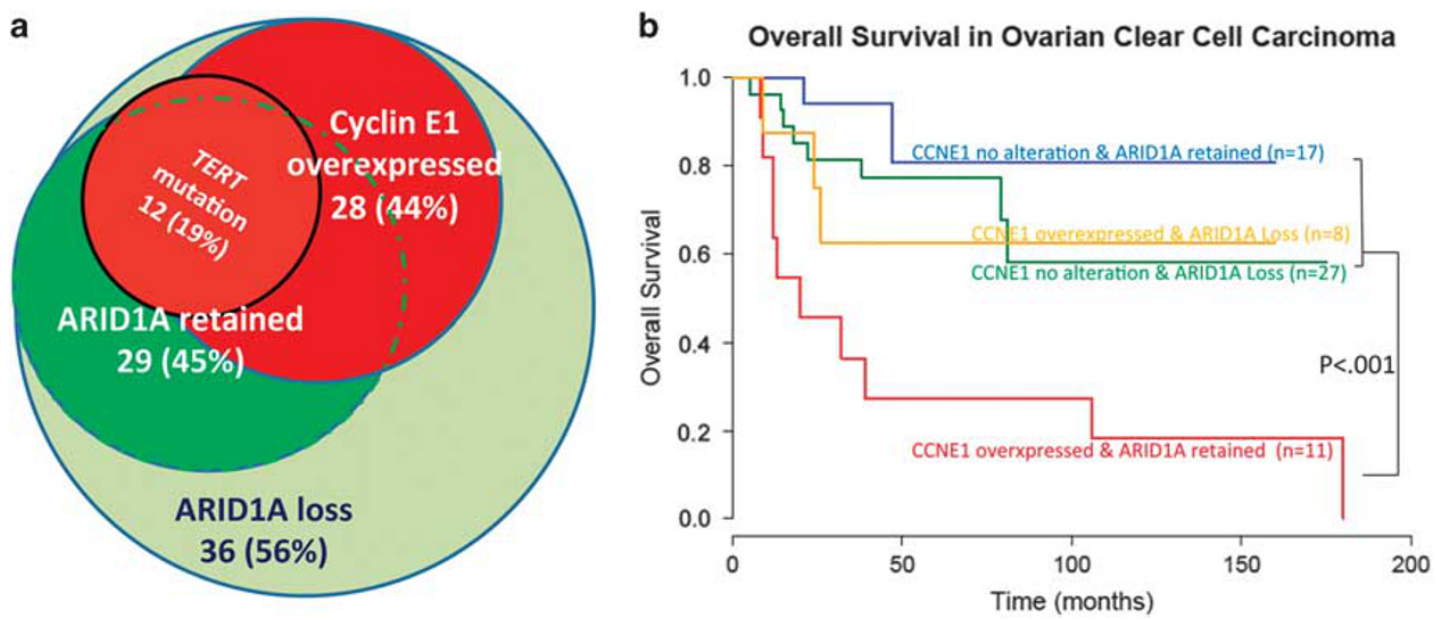

Figure 2 The relationship between CCNE1, ARID1A, and hTERT mutation status in 64 and 65 overlapping patients with clear cell carcinomas (a). Patients with both CCNE1 overexpression and ARID1A retention have the worst survival. No association was found between overall survival (either all patients or stage 1 patients) and hTERT mutation or ARID1A loss (b).

suggests that a subset of clear cell carcinomas may be molecularly close to type II ovarian cancer. The absence of CCNE1 gene copy-number gain and the lack of hTERT promoter mutations in ovarian endometrioid carcinoma ${ }^{3,21}$ further indicate distinct molecular pathways that are involved in the development of ovarian clear cell and endometrioid carcinomas.

The amplification and subsequent overexpression of CCNE1 have been reported as one of the major molecular mechanisms that promote the progression of ovarian high-grade serous carcinoma, the most 
common and aggressive type of ovarian cancer. ${ }^{19,23}$ Here we demonstrated that clear cell carcinomas also harness this molecular pathway to promote aggressive behaviour, and that women whose clear cell carcinomas display CCNE1 copy-number gain or overexpression exhibit a dismal outcome compared with those without CCNE1. It should be noted that the FISH signals of the CCNE1 locus that were observed in clear cell carcinomas were mostly discrete countable signals, probably representing a double minute type amplicon, whereas the CCNE1 amplification detected in high-grade serous carcinoma often exhibited as aggregated clusters of signals, most likely representing a homogenously staining region type amplicon. The above findings suggest that different mechanisms are involved. Cyclin E1 has been established as a critical factor in the regulation of cell cycle progression, and overexpression of cyclin E1 has been reported to result in genomic instability. Cyclin E1 can inappropriately initiate DNA replication and centrosome duplication, which leads to chromosomal instability, aneuploidy, and eventually tumorigenesis. ${ }^{24,25}$ As the prognosis for advanced-stage ovarian clear cell carcinoma is poor, ${ }^{26,27}$ there is an unmet need to identify clear cell carcinoma patients who are at an increased risk for the development of progressive diseases despite intensive platinum-based chemotherapy. Remarkably, the copy-number gain can occur in the early stages of the disease and it can also predict poor disease outcome. In agreement with this finding, we also found that either immunohistochemistry or FISH was similar in performance to identify patients with better and worse survival. Furthermore, those ovarian clear cell carcinomas with CCNE1 upregulation (gene copy-number gain and/or overexpression) and ARID1A retention is associated with worse disease outcome even though their morphological features are indistinguishable from those of the other clear cell carcinoma types.

Clinically, women presenting stage I ovarian clear cell carcinomas may be candidates for chemotherapy because some of them may progress even after their tumors are resected. Therefore, it would be of great interest for the future clinical studies to determine whether cyclin E1 and ARID1A immunohistochemistry would help identify those with aggressive clinical behavior and they may warrant a more intensive therapy. For example, clear cell carcinomas exhibiting CCNE1 upregulation and ARID1A expression may benefit from target-based therapy. Future research is needed to comprehensively elucidate the molecular landscape that accounts for this dismal outcome in this subset of clear cell carcinomas.

Although this study provides several new insights into the pathogenesis of clear cell carcinoma and offers novel potential biomarkers to identify a subset of clear cell carcinomas with the most aggressive clinical behavior, several limitations of this study should be discussed. First, all the tumors that were analyzed in the current study were derived from patients from Japan, where the percentages of clear cell carcinoma are higher than in Western countries. It remains unclear whether the pathogenesis and molecular landscape differ among individuals of various ethnic backgrounds because the previous comprehensive genomic analysis was not performed in this population. Second, as discussed above, we did not observe high CCNE1 DNA copy-number gain (so-called 'homogenously staining region type amplicon') in this set of clear cell carcinomas as in uterine serous carcinomas. ${ }^{15,19}$ The CCNE1 copynumber gain in some of the tumors may be attributed to a high polysomy event rather than to the actual duplication of the discrete CCNE1 locus. However, we observed a strong correlation between DNA copynumber gain and immunoreactivity in clear cell carcinomas, which suggests that CCNE1 copynumber gain has a biological significance instead of just a bystander effect. Third, in this study, we found that clear cell carcinomas with either overexpression of CCNE1 or CCNE1 copy-number gain tend to not harbor hTERT promoter (activating) mutations. Why this occurs is not known, but one explanation is that cyclin E1 upregulation that is due to either increased gene copy number or transcriptional activation may participate in the maintenance of telomere length through hTERT promoter activation. This is because hTERT expression can be reactivated in tumors that harbor hTERT promoter activating mutations. ${ }^{28,29}$ Indeed, it has been reported that, in breast cancer, the highest telomerase activity was found in carcinomas with cyclin E1 overexpression. ${ }^{30}$ Further studies are required to determine whether cyclin E1 also contributes to the maintenance of telomeres in ovarian clear cell carcinomas and to determine the mechanisms involved.

In summary, we found that CCNE1 copy-number gain and overexpression occurred in ovarian clear cell carcinoma, but not in ovarian endometrioid or seromucinous carcinomas. CCNE1 copy-number gain and overexpression showed a high tendency towards mutual exclusivity with hTERT promoter mutations, but not with the loss of ARID1A expression. CCNE1 copy-number gain and overexpression confer a poor prognosis, especially in the subset with ARID1A retention. These results suggest the importance of CCNE1 in the progression of ovarian clear cell carcinoma and support cyclin E1 as a possible therapeutic target in ovarian clear cell carcinoma.

\section{Acknowledgments}

This project was supported, in part, by SRF, MEXT (S-001), the National Cancer Center Research and Developing Fund, and A-MED, from Japan, and Richard W. TeLinde GynecologicPathology Program at Johns Hopkins Medical Institutions. 


\section{Disclosure/conflict of interest}

The authors declare no conflict of interest.

\section{References}

1 Kurman RJ, Shih Ie M. The dualistic model of ovarian carcinogenesis: revisited, revised, and expanded. Am J Pathol 2016;186:733-747.

2 Yamaguchi K, Mandai M, Toyokuni S, et al. Contents of endometriotic cysts, especially the high concentration of free iron, are a possible cause of carcinogenesis in the cysts through the iron-induced persistent oxidative stress. Clin Cancer Res 2008;14:32-40.

$3 \mathrm{Wu}$ RC, Wang TL, Shih IM. The emerging roles of ARID1A in tumor suppression. Cancer Biol Ther 2014;15: 655-664.

4 Kobel M, Kalloger SE, Carrick J, et al. A limited panel of immunomarkers can reliably distinguish between clear cell and high-grade serous carcinoma of the ovary. Am J Surg Pathol 2009;33:14-21.

5 Fadare O, Zhao C, Khabele D, et al. Comparative analysis of Napsin A, alpha-methylacyl-coenzyme A racemase (AMACR, P504S), and hepatocyte nuclear factor 1 beta as diagnostic markers of ovarian clear cell carcinoma: an immunohistochemical study of 279 ovarian tumours. Pathology 2015;47:105-111.

6 Kandalaft PL, Gown AM, Isacson C. The lung-restricted marker napsin A is highly expressed in clear cell carcinomas of the ovary. Am J Clin Pathol 2014;142: 830-836.

7 Skirnisdottir I, Bjersand K, Akerud H, et al. Napsin A as a marker of clear cell ovarian carcinoma. BMC Cancer 2013;13:524.

8 Guan B, Rahmanto YS, Wu RC, et al. Roles of deletion of Arid1a, a tumor suppressor, in mouse ovarian tumorigenesis. J Natl Cancer Inst 2014;106:dju 146.

9 Chandler RL, Damrauer JS, Raab JR, et al. Coexistent ARID1A-PIK3CA mutations promote ovarian clear-cell tumorigenesis through pro-tumorigenic inflammatory cytokine signalling. Nat Commun 2015;6:6118.

10 Jones S, Wang TL, Shih Ie M, et al. Frequent mutations of chromatin remodeling gene ARID1A in ovarian clear cell carcinoma. Science 2010;330:228-231.

11 Keyomarsi K, Tucker SL, Bedrosian I. Cyclin E is a more powerful predictor of breast cancer outcome than proliferation. Nat Med 2003;9:152.

12 Nakayama N, Nakayama K, Shamima Y, et al. Gene amplification CCNE1 is related to poor survival and potential therapeutic target in ovarian cancer. Cancer 2010;116:2621-2634.

13 Rosen DG, Yang G, Deavers MT, et al. Cyclin E expression is correlated with tumor progression and predicts a poor prognosis in patients with ovarian carcinoma. Cancer 2006;106:1925-1932.

14 Kuhn E, Wu RC, Guan B, et al. Identification of molecular pathway aberrations in uterine serous carcinoma by genome-wide analyses. J Natl Cancer Inst 2012;104: 1503-1513.

15 Kuhn E, Bahadirli-Talbott A, Shih Ie M. Frequent CCNE1 amplification in endometrial intraepithelial carcinoma and uterine serous carcinoma. Mod Pathol 2014;27:1014-1019.

16 Cassia R, Moreno-Bueno G, Rodriguez-Perales S, et al. Cyclin E gene (CCNE) amplification and hCDC4 mutations in endometrial carcinoma. J Pathol 2003;201: 589-595.

17 Bedrosian I, Lu KH, Verschraegen C, et al. Cyclin E deregulation alters the biologic properties of ovarian cancer cells. Oncogene 2004;23:2648-2657.

18 Karst AM, Jones PM, Vena $\mathrm{N}$, et al. Cyclin E1 deregulation occurs early in secretory cell transformation to promote formation of fallopian tube-derived high-grade serous ovarian cancers. Cancer Res 2014;74: 1141-1152.

19 Kuhn E, Wang TL, Doberstein K, et al. CCNE1 amplification and centrosome number abnormality in serous tubal intraepithelial carcinoma: further evidence supporting its role as a precursor of ovarian high-grade serous carcinoma. Mod Pathol (e-pub ahead of print 22 July 2016; doi:10.1038/modpathol.2016.101).

20 Guan B, Wang TL, Shih Ie M. ARID1A, a factor that promotes formation of SWI/SNF-mediated chromatin remodeling, is a tumor suppressor in gynecologic cancers. Cancer Res 2011;71:6718-6727.

$21 \mathrm{Wu}$ RC, Ayhan A, Maeda D, et al. Frequent somatic mutations of the telomerase reverse transcriptase promoter in ovarian clear cell carcinoma but not in other major types of gynaecological malignancy. J Pathol 2014;232:473-481.

22 Ayhan A, Mao TL, Seckin T, et al. Loss of ARID1A expression is an early molecular event in tumor progression from ovarian endometriotic cyst to clear cell and endometrioid carcinoma. Int J Gynecol Cancer 2012;22:1310-1315.

23 Nakayama K, Nakayama N, Jinawath N, et al. Amplicon profiles in ovarian serous carcinomas. Int J Cancer 2007;120:2613-2617.

24 Pundir S, Vu HY, Solomon VR, et al. VR23: a quinoline-sulfonyl hybrid proteasome inhibitor that selectively kills cancer via cyclin E-mediated centrosome amplification. Cancer Res 2015;75:4164-4175.

25 Kawamura K, Izumi $\mathrm{H}$, Ma $\mathrm{Z}$, et al. Induction of centrosome amplification and chromosome instability in human bladder cancer cells by p53 mutation and cyclin E overexpression. Cancer Res 2004;64:4800-4809.

26 Pather S, Quinn MA. Clear-cell cancer of the ovary-is it chemosensitive? Int J Gynecol Cancer 2005;15: 432-437.

27 Takano M, Sugiyama T, Yaegashi N, et al. Low response rate of second-line chemotherapy for recurrent or refractory clear cell carcinoma of the ovary: a retrospective Japan Clear Cell Carcinoma Study. Int J Gynecol Cancer 2008;18:937-942.

28 Horn S, Figl A, Rachakonda PS, et al. TERT promoter mutations in familial and sporadic melanoma. Science 2013;339:959-961.

29 Huang FW, Hodis E, Xu MJ, et al. Highly recurrent TERT promoter mutations in human melanoma. Science 2013;339:957-959.

30 Landberg G, Nielsen NH, Nilsson P, et al. Telomerase activity is associated with cell cycle deregulation in human breast cancer. Cancer Res 1997;57:549-554. 